\title{
Note sur quelques séries de puissances trouvées dans la théorie de la fonction gamma.
}

\author{
(Par Niels Nielsen, à Copenhague.)
}

I. Sḱries de puissaxces obtenues pour $\Gamma(1+x)$ et pour $\frac{1}{1(1+x)}$.

\section{$P_{\text {osons pour abréger }}$}

nous aurons, pour $n=1$,

$$
\Gamma(x)=e^{\gamma(x)},
$$

$$
-\gamma^{(1)}(x)=-D_{x} \log \Gamma(x)=C+\sum_{s=0}^{s \overline{\overline{\mathbf{y}}}}\left(\frac{1}{x+s}-\frac{1}{s+1}\right)=s_{1}(x)\left(^{*}\right),
$$

où $C$ désigne la constante $d^{\prime} E u L E R$, et plus généralement, pour $n \geq 2$,

$$
\frac{(-1)^{n}}{(n-1) !} \cdot y^{\prime(n)}(x)=s_{n}(x)=\frac{1}{x^{n}}+\frac{1}{(x+1)^{n}}+\frac{1}{(x+2)^{n}}+\cdots
$$

de plus nous posons

$$
s_{1}=C=s_{\mathrm{t}}(1), \quad s_{n}=s_{n}(1)=\frac{1}{1^{n}}+\frac{1}{2^{n}}+\frac{1}{3^{n}}+\cdots
$$

On sait que $\Gamma(1+x)$ est holomorphe aux environs du point $x=0$ et que la série de puissances correspondante, savoir la série

$$
\Gamma(1+x)=1+c_{1} x+c_{2} x^{2}+c_{3} x^{3}+\cdots
$$

a son rayon de convergence égal à un.

$(*)$ On voit que cette définition de $s_{1}(x)$ est un peu différente de celle que $j$ 'ai appliquée dans mon mémoirè précédent dans ce même volume, p. 189. 
Pour déterminer l'expression développée du coefficient général a figurant au second membre de (4) appliquons cette formule due it feu M. R. Hoppe (*)

$$
D_{x}^{n} F(y)=\sum_{k=1}^{k=i} \frac{1}{k !} \cdot T(x) F^{k, n}(y)
$$

où $y=f(x)$, et où nous avons posé pour abréger

$$
T^{k, n}(y)=\sum_{s=0}^{s=k}(-1)^{s}\left(\begin{array}{l}
k \\
s
\end{array}\right) y^{s} D_{x}^{n}\left(y^{k-s}\right)
$$

Or, dans le cas particulier qui nous occupe ici, où $F(y)=e^{y}$, cette for.mule g'énérale se simplifie beaucoup. Fn effet, il est évident que l'expression $T^{h, u}(x)$ ne peut pas contenir la fonction $y$ elle-même; e'est-à-dire que la formule polynomiale symbolique pour l'évaluation de

donnera dans ce cas

$$
D_{x}^{n}\left(y_{1} y_{z} y_{3} \ldots y_{k}\right)
$$

$$
T(y)=n ! \Sigma \frac{y^{\left(r_{1}\right)} y^{\left(r_{k}\right)} \ldots y^{\left(r_{k}\right)}}{r_{1} ! r_{2} ! \ldots r_{k} !}
$$

où la sommation qui figure au second membre doit être étendue à toutes les combinaisons possibles des $k$ nombres positifs entiers

$$
r_{1}, r_{2}, r_{3}, \ldots r_{k}
$$

qui satisfont à cette seule condition

$$
r_{1}+r_{2}+r_{3}+\cdots+r_{t}=n \text {. }
$$

Posons maintenant $y==\gamma(x)$, nous aurous, en vertu de (2) et $\left(2^{\text {bis }}\right)$,

$$
T^{k, n}(y)=(-1)^{n} n ! \Sigma \frac{s_{r_{1}}(x) s_{r_{2}}(x) \ldots s_{r_{k}}(x)}{r_{1} \cdot r_{2} \cdot r_{3} \ldots r_{k}}=(-1)^{n} n ! V^{k, n}(x)
$$

où nous avons posé pour abréger

$$
V(x)=\Sigma \frac{s_{r_{1}}(x) s_{r_{2}}(x) \ldots s_{r_{k}}(x)}{r_{1} \cdot r_{z} \cdot r_{3} \ldots r^{*} r_{2}}
$$

et nous obtenons finalement cette formule générale

$$
\Gamma^{(n)}(x)=(-1)^{n} n ! \Gamma(x) \cdot \sum_{k=1}^{k=n} \frac{1}{k !} \cdot V^{k, n}(x)
$$

(*) Théorie der höheren Differentialciotienten, Leipsic, 1845. - Voir aussi: ScHLöxncu, Compendirm der höheren Analysis, t. II, p. 6, Brunswiek, 1870. 
ce qui donnera pour le coefficient $c_{n}$ cette expression

$$
C_{n}=(-1)^{n} \cdot \sum_{k=1}^{k=n} \frac{1}{k !} \cdot V(1),
$$

où nous avons par conséquent

$$
V(1)=\Sigma \frac{s_{r_{1}} s_{r_{0}} \ldots s_{r_{k}}}{r_{i_{1}, r_{2} \ldots r_{k}}}
$$

Considérons ensuite cette autre fonction

$$
\overline{\Gamma(1+x)}=\frac{1}{x \Gamma(x)}=\frac{1}{x} \cdot e^{-\gamma(x)},
$$

la formule de Leinnitz donnera cette formule différentielle générale

$$
D_{x}^{n}\left(\frac{1}{\Gamma(1+x)}\right)+\frac{n}{x} \cdot D_{x}^{n-1}\left(\frac{1}{\Gamma(1+x)}\right)=\frac{1}{x} \cdot D_{x}^{n} e^{-z(x)} .
$$

En effet multiplions par $x$ les deux membre de (7), puis différentions $n$ fois par rapport à $x$, une nouvelle division par cette variable nous conduira jmmédiatement à (8).

Appliquons maintenant la méthode que nous venons d'indiquer, nous aurons sans peine

$$
\begin{gathered}
D_{x}^{n}\left(\frac{1}{\Gamma(1+x)}\right)_{x=1}+n D_{x}^{n-1}\left(\frac{1}{\Gamma(1+x)}\right)_{x=1}= \\
=(-1)^{n} n ! \sum_{k=1}^{k=n} \frac{(-1)^{k}}{k !} \cdot V^{k, n}(1)
\end{gathered}
$$

ce qui donnera pour les coefficients généraux de cette série de puissances

$$
\frac{1}{\Gamma(2+x)}=1+d_{1} x+d_{2} x^{2}+d_{3} x^{3}+\cdots
$$

cette relation générale

$$
d_{n}+d_{n-1}=(-1)^{n} \cdot \sum_{k=1}^{k=\bar{\Sigma}^{n}} \frac{(-1)^{k}}{k !} \cdot V^{k, n}(1) .
$$

Cela posé, multiplions par $(x+1)$ les deux membres de $(\alpha)$, nous aurons, en vertu de $(\beta)$, pour le coefficient général de cette série de puissances qui est valable dans toute l'étendue du plan des $x$, savoir la série

$$
\frac{1}{\Gamma(1+x)}=1+y_{1} x+\gamma_{2} x^{2}+\gamma_{3} c^{3}+\cdots,
$$


cette expression

$$
\left.\gamma_{n}=(-1)^{n} \cdot \sum_{k=1}^{k=1} \frac{(-1)^{k}}{k !} \cdot V_{! 1}^{k, n} 1\right)
$$

où $V^{k, n}(1)$ désigne la quantité définie à l'aide de la formule $\left(6^{\text {bis }}\right)$, de sorte que nous avons démontré ces deux théorèmes dont le premier et bien connu, tandis que le second semble être nouveau:

I. Les coefficients généraux $c_{n}$ et $\gamma_{n}$ des séries de puissances (4) et (9) s'expriment sous forme des polynomes entier's et homogènẹs du dégré $n$ des nomỏres $s_{1} s_{2} \ldots s_{n}$, si nous supposons $s_{r}$ du dégré $r ;$ les coefficients de ces polynomes sont des nombres rationnels.

II. L'expression susdite de $\gamma_{n}$ peut être déduite de celle obtenue pour: $c_{n}$ en $y$ posant simplement $-s_{r}$ au lieu de $s_{r}$.

Les expressions développées (6) et $(9$ is $)$ pour $c_{n}$ et $y_{n}$ qui sont nouvelles peut-être ne sont guère convenables pour un calcul numérique; pour obtenir les valeurs approchées des coefficients susdits ont peut partir de certaines formules récursives, comme l'a fait $\mathbf{M}$. Bourquer $\left({ }^{*}\right)$.

II. SḱRIES DE PUISSANCES ObTENUES POUR $B\left(\frac{x+1}{2}, \frac{1}{2}\right)$ ET POUR $x . B\left(\frac{x}{2}, \frac{1}{2}\right)$.

Il est bien remarquable que les résultats précédents peuvent être étendus à un cas particulier de la fonction beta aussi. En effet, posons

$$
B\left(\frac{x}{2}, \frac{1}{2}\right)=\frac{\Gamma\left(\frac{x}{2}\right) \Gamma\left(\frac{1}{2}\right)}{\Gamma\left(\frac{x+1}{2}\right)}=e^{\beta(x)},
$$

nous aurons, en vertu de (2),

$$
-\beta^{(1)}(x)=\frac{1}{2}\left(s_{1}\left(\frac{x}{2}\right)-s_{1}\left(\frac{x+1}{2}\right)\right)=\sigma_{1}(x),
$$

où nous avons posé pour abréger

$$
\sigma_{1}(x)=\frac{1}{x}-\frac{1}{x+1}+\frac{1}{x+2}-\frac{1}{x+3}+\cdots
$$

(*) Acta Mathematica, t. II, p. 288, p. $291 ; 1884$. 
et plus généralement

$$
\sigma_{r}(x)=\frac{(-1)^{r}}{(r-1) !} \cdot \beta^{(r)}(x)=\frac{1}{x^{r}}-\frac{1}{(x+1)^{r}}+\frac{1}{(x+2)^{r}}-\cdots
$$

Pour l'argument 1 nous posons encore pour abréger

ce qui donnera

$$
\sigma_{r}(1)=\sigma_{r}=\frac{1}{1^{r}}-\frac{1}{2^{r}}+\frac{1}{3^{r}}-\frac{1}{4^{r}}+\cdots,
$$

et plus généralement, pour $n \geqq 2$,

$$
\sigma_{n}=\frac{2^{n-1}-1}{2^{n-1}} \cdot s_{n} \text {. }
$$

Cela posé, considérons cette série de puissances

$$
\frac{1}{2} \cdot B\left(\frac{x+1}{2}, \frac{1}{2}\right)=\frac{\pi}{2}+b_{1} x+b_{2} x^{2}+b_{3} x^{3}+\cdots, \quad|x|<1,
$$

nous aurons

$$
2 b_{n}=\frac{1}{n !} \cdot\left(D_{x}^{n} B\left(\frac{x}{2}, \frac{1}{2}\right)\right)_{x=1}
$$

de sorte que le même procédé que nous venons d'appliquer sur la fonction gamma donnera ici pour le coefficient $b_{n}$ cette expression

$$
b_{n}=(-1)^{n} \cdot \frac{\pi}{2} \cdot \sum_{k=1}^{k=12} \frac{1}{k !} \cdot U^{k, n}(1)
$$

où nous avons posé pour abréger

$$
\begin{gathered}
U^{k \cdot n}(\mathrm{j})=\mathbf{\Sigma} \frac{\sigma_{r_{1}} \sigma_{r_{2}} \ldots \sigma_{r_{k}}}{r_{1} \cdot r_{2} \ldots r_{k}}, \\
r_{1}+r_{2}+r_{3}+\cdots+r_{k}=n
\end{gathered}
$$

Appliquons ensuite cette formule fondamentale $\Gamma(1+x)=x . \Gamma(x)$, nous aurons de même, en vertu de $(10)$,

$$
B\left(\frac{x+1}{2}, \frac{1}{2}\right)=\frac{2 \pi}{x} \cdot \frac{1}{B\left(\frac{x}{2}, \frac{1}{2}\right)}=\frac{2 \pi}{x} \cdot e^{-\beta(x)},
$$

ce qui donnera cette autre formule analogue à (8)

$$
D_{x}^{n} B\left(\frac{x+1}{2}, \frac{1}{2}\right)+\frac{n}{x} \cdot D_{x}^{n-1} B\left(\frac{x+1}{2}, \frac{1}{2}\right)=\frac{2 \pi}{x} \cdot D_{x}^{n} e^{-\beta(x)},
$$


d'où pour $x=1$

$$
\begin{gathered}
D_{x}^{n} B\left(\frac{x+1}{2}, \frac{1}{2}\right)_{x=1}+n D_{x}^{n-1} B\left(\frac{x-1}{2}, \frac{1}{2}\right)_{x=1}= \\
=(-1)^{n} \cdot 2 \cdot n ! \sum_{k=1}^{k=1} \frac{(-1)^{k}}{k !} \cdot U^{\prime}(1)
\end{gathered}
$$

où $U^{k, n}(1)$ désigne la quantité que nous venons de définir à l'aide de $\left(12^{\text {bis }}\right)$.

Considérons maintenant cette autre série de puissances

$$
\begin{aligned}
& \frac{1}{2} \cdot B\left(\frac{x+2}{2}, \frac{1}{2}\right)=1+d_{1} x+d_{2} x^{2}+d_{3} x^{3}+\cdots \\
& \text { coefficients } d_{n} \text { doivent satisfaire à cette condition } \\
& \qquad d_{n}+d_{n-1}=(-1)^{n} \cdot \sum_{k=1}^{k=n} \frac{(-1)^{k}}{k !} \cdot U^{k, n}(1) .
\end{aligned}
$$

Cela posé, cette formule fondamentale

$$
B\left(\frac{x+2}{2}, \frac{1}{2}\right)=\frac{x}{x+1} B\left(\frac{x}{2}, \frac{1}{2}\right)
$$

donnera, si nous multiplions par $(x+1)$ les deux membres de $(\alpha)$, cette nouvelle série de puissances

$$
\frac{x}{2} \cdot B\left(\frac{x}{2}, \frac{1}{2}\right)=1+\beta_{1} x+\beta_{2} x^{2}+\beta_{3} x^{3}+\cdots, \quad|x|<2,
$$

dont le coefficient général se détermine par cette formule

$$
\beta_{n}=(-1)^{n} \cdot \sum_{k=1}^{k=\bar{z}^{n}} \frac{(-1)^{k}}{k !} \cdot U^{k, n}(1)
$$

de sorte que nous avons démontré ces deux théorèmes qui semblent être nouveaux:

III. Le coefficient $\beta_{n}$ de la série de puissances (13) peut être formé si nous remplace, dans l'expression obtenue pour $\gamma_{n}, s_{r}$ par $\sigma_{r}$.

IV. Le coefficient $b_{n}$ de la série de puissances (11) peut être déduit en multipliant par $\frac{\pi}{2}$ l'expression obtenue de $\beta_{n}$ en $y$ posant $-\sigma_{r}$ au lieu de $\sigma$, .

Il est évident que les expressions développées que nous venons de donner pour les coefficients généraux $b_{n}$ et $\beta_{n}$ ne sont guère commodes pour un calcul numérique. Cependant, il est très facile de représenter les deux coef- 
ficient susdits sous forme d'une série numérique qui peut être calculée approximativement sans peine quand l'indice $n$ et assez grand. Pour déduire de telles séries numériques appliquons cette formule bien connue

d'ou

$$
\int_{0}^{1} t^{x-1}\left(1-t^{2}\right)^{-\frac{1}{2}} d t=\frac{1}{2} \cdot B\left(\frac{x}{2}, \frac{1}{2}\right), \quad \Re(x)>0,
$$

$$
\int_{0}^{1} \frac{(\log t)^{n}}{\sqrt{1-t^{2}}} d t=\frac{1}{2} \cdot\left(D_{x}^{n} B\left(\frac{x}{2}, \frac{1}{2}\right)\right)_{x=1} .
$$

Cela posé, la formule du binome donnera

$$
\frac{1}{\sqrt{1-t^{2}}}=1+\frac{1}{2} \cdot t^{2}+\frac{1.3}{2 \cdot 4} \cdot t^{4}+\frac{1.3 \cdot 5}{2 \cdot 4 \cdot 6} \cdot t^{6}+\cdots
$$

d'où, en vertu de cette formule intégrale, où $r$ désigne un entier non négatif

$$
\int_{0}^{1} t^{x-1}(\log t)^{r} d t=\frac{(-1)^{r} r !}{x^{2}+1}, \quad \Re(x)>0
$$

et en appliquant $(\delta)$, cette expression nouvelle de $b_{n}$

$$
(-1)^{n} b_{n}=\frac{1}{1^{n+1}}+\frac{1}{2} \cdot \frac{1}{3^{n+1}}+\frac{1.3}{2.4} \cdot \frac{1}{5^{n+1}}+\frac{1.3 \cdot 5}{2.4 \cdot 6} \cdot \frac{1}{7^{n+4}}+\cdots
$$

Nous aurons de même

$$
\begin{gathered}
\frac{(-1)^{n}}{n !} \cdot \int_{0}^{1}\left(\frac{1}{\sqrt{1-t^{2}}}-1\right) \frac{(\log t)^{n}}{t} d t= \\
=\frac{1}{2} \cdot \frac{1}{2^{n+1}}+\frac{1.3}{2 \cdot 4} \cdot \frac{1}{4^{n+1}}+\frac{1.3 \cdot 5}{2 \cdot 4 \cdot 6} \cdot \frac{1}{6^{n+1}}+\cdots
\end{gathered}
$$

et, en vertu de (y),

$$
\int_{0}^{1}\left(\frac{1}{\sqrt{1-t^{2}}}-1\right) t^{x-1} d t=\frac{1}{2} \cdot B\left(\frac{x}{2}, \frac{1}{2}\right)-\frac{1}{x}
$$

ce qui donnera, en appliquant la série de puissances (13),

$$
\int\left(\frac{1}{\sqrt{1-t^{2}}}-1\right) t^{x-1} d t=\beta_{1}+\beta_{2} x+\beta_{3} x^{2}+\beta_{4} x^{3}+\cdots
$$


de sorte que nous obtiendrons à l'aide de la série numérique $(\varepsilon)$, cette représentation nouvelle $d u$ coefficient $\beta_{n}$ :

$$
(-1)^{n-1} \beta_{n}==\frac{1}{2} \cdot \frac{1}{2^{n}}+\frac{13}{2.4} \cdot \frac{1}{4^{n}}+\frac{1.3 .5}{2.4 .6} \cdot \frac{1}{6^{n}}+\cdots
$$

Les formules (14) (15) donnent précisément les représentations nouvelles des coefficients $b_{n}$ et $\beta_{n}$ que nous avions nous proposé de développer.

Quant aux séries numériques

$$
\begin{aligned}
& A_{n}=\frac{1}{1^{n}}+\frac{1}{2} \cdot \frac{1}{3^{n}}+\frac{1.3}{2.4} \cdot \frac{1}{5^{n}}+\frac{1.3 .5}{2.4 .6} \cdot \frac{1}{7^{n}}+\cdots \\
& B_{n}=\frac{1}{2} \cdot \frac{1}{2^{n}}+\frac{1.3}{2.4} \cdot \frac{1}{4^{n}}+\frac{1.3 .5}{2.4 .6} \cdot \frac{1}{6^{n}}+\cdots
\end{aligned}
$$

nous avons, en vertu des théorèmes III et IV, cette proposition remarquable:

V. La somme de la série numérique $B_{n}$ est égale à un polynome entier à coefficients rationnels de $\sigma_{1}, \sigma_{2}, \ldots, \sigma_{n}$ et homogène du dégré $n$ dans ces nombres, si nous supposons $\sigma_{r} d u$ dégré $r$. La somme de la série $A_{n}$, au contraire, peut être déduite en multipliant par $-\frac{\pi}{2}$ l'expression obtenue de $B_{n+1}$ en $y$ posant $-\sigma_{r}$ au lieu de $\sigma_{r}$.

Nous aurons par exemple

$$
\begin{aligned}
& B_{1}=\sigma_{1}=\log 2 \\
& A_{2}=\frac{\pi}{2} \cdot \log 2
\end{aligned}
$$

ou, ce qui vaut autant, cette formule eulérienné

$$
-\int^{\frac{\pi}{2}} \log \sin \varphi d \varphi=\frac{\pi}{2} \cdot \log 2
$$

en effet, pour déduire cette formule nous n'avons qu'à mettre dans $(\delta) n==1$ et $t=\sin \varphi$.

Copenhague, le 25 avril 1908. 